\title{
CHARACTERIZATIONS OF PLURISUBHARMONIC FUNCTIONS
}

\author{
FUSHENG DENG, JIAFU NING, AND ZHIWEI WANG
}

\begin{abstract}
AвSTRACT. We give characterizations of (quasi-)plurisubharmonic functions in terms of $L^{p}$-estimates of $\bar{\partial}$ and $L^{p}$-extensions of holomorphic functions.
\end{abstract}

\section{INTRODUCTION}

In the fundamental work [12], Hörmander established a systematic $L^{2}$-theory for $\bar{\partial}$ operator. In [14], Ohsawa and Takegoshi proved an extension theorem for $L^{2}$ holomorphic functions, now known as Ohsawa-Takegoshi extension theorem. Hörmander's $L^{2}$-theory for $\bar{\partial}$ and Ohsawa-Takegoshi extension theorem are of fundamental importance in several complex variables and have terrific applications in algebraic geometry.

In both Hörmander's $L^{2}$-theory for $\bar{\partial}$ and Ohsawa-Takegoshi extension theorem, plurisubharmonic functions are used as weights and play a key role. It is natural to ask if plurisubharmonic functions are the only choice for weights in both theories? The aim of the present paper is to answer this question affirmatively and give characterizations of (quasi-)plurisubharmonic functions in terms of $L^{p}$-estimates of $\bar{\partial}$ and $L^{p}$-extensions of holomorphic functions, which can be roughly understood as converses of Hörmander's $L^{2}$ estimate of $\bar{\partial}$ and Ohsawa-Takegoshi extension theorem. To state the results, we first introduce some notions.

Definition 1.1. Let $\phi: D \rightarrow[-\infty,+\infty)$ be an upper semi-continuous function on a domain $D$ in $\mathbb{C}^{n}$. We say that:

(1) $\phi$ satisfies the optimal $L^{p}$-estimate property if for any $\bar{\partial}$-closed smooth $(0,1)$-form $f$ on $D$ with compact support and any smooth strictly plurisubharmonic function $\psi$ on $D$, the equation $\bar{\partial} u=f$ can be solved on $D$ with the estimate

$$
\int_{D}|u|^{p} e^{-\phi-\psi} \leq \int_{D}|f|_{i \partial \partial \bar{\partial} \psi}^{p} e^{-\phi-\psi}
$$

provided that the right hand side is finite.

2010 Mathematics Subject Classification. 32W05, 32U05, 32T99, 32A70.

Key words and phrases. Plurisubharmonic functions, Hörmander's $L^{2}$-estimate, Ohsawa-Takegoshi extension theorem, characterization of plurisubharmonicity. 
(2) $\phi$ satisfies the multiple coarse $L^{p}$-estimate property if for any $m \geq 1$, any $\bar{\partial}$-closed smooth $(0,1)$-form $f$ on $D$ with compact support, and any smooth strictly plurisubharmonic function $\psi$ on $D$, the equation $\bar{\partial} u=f$ can be solved on $D$ with the estimate

$$
\int_{D}|u|^{p} e^{-m \phi-\psi} \leq C_{m} \int_{D}|f|_{i \partial \partial \bar{\partial} \psi}^{p} e^{-m \phi-\psi},
$$

provided that the R.H.S is finite, where $C_{m}$ are constants such that $\lim _{m \rightarrow \infty} \log C_{m} / m=0$.

(3) $\phi$ satisfies the optimal $L^{p}$-extension property if for any $z \in D$ with $\phi(z) \neq-\infty$, and any holomorphic cylinder $P$ with $z+P \subset D$, there is a holomorphic function $f$ on $z+P$ such that $f(z)=1$ and

$$
\frac{1}{\mu(P)} \int_{z+P}|f|^{p} e^{-\phi} \leq e^{-\phi(z)}
$$

where $\mu(P)$ is the volume of $P$ with respect to the Lebesgue measure. (Here by a holomorphic cylinder we mean a domain of the form $A\left(P_{r, s}\right)$ for some $A \in U(n)$ and $r, s>0$, with $\left.P_{r, s}=\left\{\left(z_{1}, z_{2}, \cdots, z_{n}\right):\left|z_{1}\right|^{2}<r^{2},\left|z_{2}\right|^{2}+\cdots+\left|z_{n}\right|^{2}<s^{2}\right\}.\right)$

(4) $\phi$ satisfies the multiple coarse $L^{p}$-extension property if for any $m \geq 1$, any $z \in D$ with $\phi(z) \neq-\infty$, there is a holomorphic function $f$ on $D$ such that $f(z)=1$ and

$$
\int_{D}|f|^{p} e^{-m \phi} \leq C_{m} e^{-m \phi(z)}
$$

where $C_{m}$ are constants such that $\lim _{m \rightarrow \infty} \log C_{m} / m=0$.

A related but different form of the optimal $L^{p}$-extension property for $p=2$ was introduced in [11], the multiple coarse $L^{p}$-extension property was introduced in [8], and the multiple coarse $L^{p}$ estimate property was introduced and named as the twisted Hörmander condition in [13] in the case that $p=2$, with a different form in dimension one case introduced in [2].

If $\phi$ is plurisubharmonic, $p=2$, and $D$ is a bounded pseudoconvex domain, then $\phi$ satisfies:

- the optimal $L^{2}$-estimate property and the multiple coarse $L^{2}$-estimate property by Hörmander's $L^{2}$-estimate of $\bar{\partial}$ (c.f. [1, Theorem 1.6.4] for an appropriate formulation);

- the multiple coarse $L^{2}$-extension property by Ohsawa-Takegoshi [14]; and

- the optimal $L^{2}$-extension property by Blocki [5] and Guan-Zhou [9, 10].

In the present paper, we will establish the converses of the above results, namely, we will show that $\phi$ must be plurisubharmonic if it satisfies one of the properties in Definition 1.1. The precise results are formulated as several theorems as follows.

Theorem 1.1. Let $D$ be a domain in $\mathbb{C}^{n}, \phi \in C^{2}(D)$. If $\phi$ satisfies the optimal $L^{2}$-estimate property, then $\phi$ is plursubharmonic on $D$.

In fact, Theorem 1.1 can be strengthened to characterize quasi-plurisubharmonicity as follows.

Theorem 1.2. Let $D$ be a domain in $\mathbb{C}^{n}, \phi \in C^{2}(D)$ and $\omega$ be a continuous real $(1,1)$-form on $D$. If for any $\bar{\partial}$-closed smooth $(0,1)$-form $f$ on $D$ with compact support and any smooth strictly 
plurisubharmonic function $\psi$ on $D$ with $i \partial \bar{\partial} \psi+\omega>0$ on $\operatorname{supp} f$, the equation $\bar{\partial} u=f$ can be solved on $D$ with the estimate

$$
\int_{D}|u|^{2} e^{-\phi-\psi} \leq \int_{D}|f|_{i \partial \bar{\partial} \psi+\omega}^{2} e^{-\phi-\psi}
$$

provided that the right hand side is finite. Then $i \partial \bar{\partial} \phi \geq \omega$ on $D$.

We prove Theorem 1.2 by connecting $\partial \bar{\partial} \phi$ with the optimal $L^{2}$-estimate property via a Bochner type identity, and then using a localization technique to produce a contradiction if $i \partial \bar{\partial} \phi \geq \omega$ is assumed to be not true.

Theorem 1.3. Let $D$ be a domain in $\mathbb{C}^{n}$ and $\phi: D \rightarrow \mathbb{R}$ be a continuous function. If $\phi$ satisfies the multiple coarse $L^{p}$-estimate property for some $p>1$, then $\phi$ is plursubharmonic on $D$.

In connection with Theorem 1.3, we remark that Berndtsson has proved the following result [1, Proposition 2.2]: let $D$ be a domain in $\mathbb{C}$ and $\phi$ be a continuous function on $D$, such that for any $m \geq 1$ and any $f \in C_{c}^{\infty}(D)$ we can solve the equation $\bar{\partial} u=f d \bar{z}$ with the estimate

$$
\int_{D}|u|^{2} e^{-m \phi} \leq C \int_{D}|f|^{2} e^{-m \phi}
$$

with $C$ a uniform constant independent of $m$, then $\phi$ is subharmonic. The method of Berndtsson depends on the fact that the $(0,1)$-Dolbeault cohomology of $\mathbb{C}$ with compact support does not vanish, and seems difficult to generalize to higher dimensions. The case of Theorem 1.3 that $p=2$ and $\phi$ is locally Hölder continuous for general dimensions was proved in [13], by showing that the multiple coarse $L^{2}$-estimate property implies the multiple coarse $L^{2}$-extension property, and then applying Theorem 1.5 in the following. The question about if the local Hölder continuity can be removed or not is proposed in [13]. Theorem 1.3 answers this question affirmatively and is proved by modifying the idea in [13].

Theorem 1.4. Let $\phi: D \rightarrow[-\infty,+\infty)$ be an upper semi-continuous function on a domain $D$ in $\mathbb{C}^{n}$. If $\phi$ satisfies the optimal $L^{p}$-extension property for some $p>0$, then $\phi$ is plurisubharmonic on $D$.

Theorem 1.4 for $n=1$ is essentially contained in [10] where it is shown that the optimal estimate of the Ohsawa-Takegoshi extension theorem implies Berndtsson's plurisubharmonic variation of relative Bergman kernels [3, 4]. The general case is proved by modifying Guan-Zhou's method.

Theorem 1.5. Let $\phi: D \rightarrow[-\infty,+\infty)$ be an upper semi-continuous function on a domain $D$ in $\mathbb{C}^{n}$. If $\phi$ satisfies the multiple coarse $L^{p}$-extension property for some $p>0$, then $\phi$ is plursubharmonic on $D$.

Theorem 1.5 was originally proved in [8], where the method is motivated by Demailly's idea on the regularization of plurisubharmonic functions [6]. In the present paper, we give a new proof of it based on Guan-Zhou's method mentioned above. 
Remark 1.1. Note that plurisubharmonicity involves exact inequalities. It is more or less reasonable to expect that sharp estimates for $\bar{\partial}$ (namely the optimal $L^{p}$-estimate property and the optimal $L^{p}$ extension property) could imply the plurisubharmonicity of $\phi$. However, it is difficult to expect that coarse estimates can encode plurisubharmonicity. The point in Theorem 1.3 and Theorem 1.5 is that we have to consider powers of the trivial line bundle with product metrics $e^{-m \phi}$, and then there is a procedure similar to taking $m$-th roots of $C_{m}$ and finally we get the exact number $1=\lim \sqrt[m]{C_{m}}$ in the limit. This is the main observation in [8].

Remark 1.2. Continuity assumption in Theorem 1.3 is in some sense optimal since the weight $\phi$ appears in integrations in both sides of the involved estimates. This condition can be weakened to semi-continuity in Theorem 1.4 and Theorem 1.5 since the related estimates involves pointwise evaluations of $\phi$. Therefore it is natural to ask whether the regularity condition on $\phi$ in Theorem 1.2 can be weakened to being continuous?

The above theorems, combining with $L^{2}$-theory of $\bar{\partial}$, imply that the four properties in Definition 1.1 are essentially equivalent for $\phi$ at least for the case that $p=2$. Considering this conclusion is built on some heavy theories, it seems interesting to find more straightforward methods to establish the equivalence of these properties.

We now recall some background and geometric meaning of plurisubharmonic functions.

A plurisubharmonic function on an open set in $\mathbb{C}^{n}$ is an upper semi-continuous function with values in $[-\infty,+\infty)$ whose restriction to any complex line is subharmonic. It turns out that the definition does not depend on the linear structure on $\mathbb{C}^{n}$ and is invariant under holomorphic coordinate changes, hence plurisubharmonic functions can be defined on complex manifolds (or even complex spaces).

Plurisubharmonic functions play very important roles in several complex variables and complex geometry. Geometrically, a basic fact is that plurisubharmonic functions are connected to positivity of curvatures of Hermitian holomorphic vector bundles.

For the line bundle case, let $L$ be a holomorphic line bundle over a complex manifold $X$ and $h$ be a Hermitian metric on $L$. Let $e$ be a holomorphic local frame of $L$ on some open set $U \subset X$. Then $\|e\|^{2}$ is a positive function on $U$ and thus can be written as $e^{-\phi}$ for some smooth function $\phi$ on $U$. Then the curvature of $(L, h)$ on $U$ can be written as $i \partial \bar{\partial} \phi$. Therefore $(L, h)$ is positive (resp. semipositive) if and only if its local weights $\phi$ are strictly plurisubharmonic (resp. plurisubharmonic). In applications, it is also very important to allow $\phi$ has singularities, we then get the so called singular Hermitian metrics on $L$ (see [7]).

The above characterization of positivity of Hermitian line bundles in terms of plurisubharmonicity can be generalized to Hermitian holomorphic vector bundles of higher rank. Let $(E, h)$ be a Hermitian holomorphic vector bundle over $X$. Then we can define some positivity-called the Griffiths positivity-of the curvature of the Chern connection on $E$. It turns out that $(E, h)$ is Griffiths negative (resp. semi-negative) if for any local nonvanishing holomorphic section $s$ of $E, \log \|s\|$ is a strictly plurisubharmonic (resp. plurisubharmonic) function, and $(E, h)$ is Griffiths positive (resp. 
semi-positive) if and only if its dual bundle $E^{*}$ with the dual metric $h^{*}$ is Griffiths negative (resp. semi-negative).

From the above discussion, we can get the conclusion that geometrically plurisubharmonicity is in some sense equivalent to Griffiths positivity of Hermitian holomorphic vector bundles.

Theorem 1.1-1.5 can be viewed as local characterizations of positive Hermitian line bundles. These results can be generalized to Hermitian holomorphic vector bundles of higher rank. However, for highlighting the key ideas in our method, we will not discuss vector bundles of higher rank in the present paper.

Acknowledgement: The authors are very grateful to Professor Xiangyu Zhou, their former advisor, for valuable discussions on related topics. The authors are partially supported by NSFC grants.

\section{Characterizations of PluRisubharmonic FUnCtions IN TERMS OF $L^{p}$-estimates of $\bar{\partial}$}

2.1. In terms of the optimal $L^{2}$-estimate property. The aim of this subsection is to prove Theorem 1.2. For convenience, we restate it here.

Theorem 2.1 (= Theorem 1.2). Let $D$ be a domain in $\mathbb{C}^{n}, \phi \in C^{2}(D)$ and $\omega$ be a continuous real $(1,1)$-form on $D$. If for any $\bar{\partial}$-closed smooth $(0,1)$-form $f$ on $D$ with compact support and any smooth strictly plurisubharmonic function $\psi$ on $D$ with $i \partial \bar{\partial} \psi+\omega>0$ on $\operatorname{supp} f$, the equation $\bar{\partial} u=f$ can be solved on $D$ with the estimate

$$
\int_{D}|u|^{2} e^{-\phi-\psi} \leq \int_{D}|f|_{i \partial \bar{\partial} \psi+\omega}^{2} e^{-\phi-\psi}
$$

provided that the right hand side is finite. Then $i \partial \bar{\partial} \phi \geq \omega$ on $D$.

We need the following lemma.

Lemma 2.2 ([12, Proposition 2.1.2]). Let $D$ be a domain in $\mathbb{C}^{n}, \phi \in C^{2}(D)$. For any $\alpha \in \mathcal{D}_{0,1}(D)$, $\alpha=\sum_{j=1}^{n} \alpha_{j} d \bar{z}_{j}$, we have

$$
\int_{D} \sum_{j, k=1}^{n} \frac{\partial^{2} \phi}{\partial z_{j} \partial \bar{z}_{k}} \alpha_{j} \bar{\alpha}_{k} e^{-\phi}+\int_{D} \sum_{j, k=1}^{n}\left|\frac{\partial \alpha_{j}}{\partial \bar{z}_{k}}\right|^{2} e^{-\phi}=\int_{D}|\bar{\partial} \alpha|^{2} e^{-\phi}+\int_{D}\left|\bar{\partial}_{\phi}^{*} \alpha\right|^{2} e^{-\phi} .
$$

We now give the proof of Theorem 2.1.

Proof. We give the proof of Theorem 2.1 in the case that $\omega$ is $C^{1}$, and the general case follows the proof by an approximation argument.

Let $\psi$ be any smooth strictly plurisubharmonic function on $D$, and

$$
\omega=i \sum_{j, k=1}^{n} g_{j \bar{k}} d z_{j} \wedge d \bar{z}_{k}
$$


By assumption, we can solve the equation $\bar{\partial} u=f$ for any $\bar{\partial}$-closed $f \in \mathcal{D}_{0,1}(D)$, with the estimate

$$
\int_{D}|u|^{2} e^{-(\phi+\psi)} \leq \int_{D}|f|_{i \partial \partial \bar{\partial} \psi+\omega}^{2} e^{-(\phi+\psi)} .
$$

For any $\alpha \in \mathcal{D}_{0,1}(D)$, we have

$$
\begin{aligned}
\left|(\alpha, f)_{\phi+\psi}\right| & =\left|(\alpha, \bar{\partial} u)_{\phi+\psi}\right| \\
& =\left|\left(\bar{\partial}_{\phi+\psi}^{*} \alpha, u\right)_{\phi+\psi}\right| \\
& \leq\|u\|_{\phi+\psi}\left\|\bar{\partial}_{\phi+\psi}^{*} \alpha\right\|_{\phi+\psi} .
\end{aligned}
$$

From Lemma 2.2, we obtain

$$
\begin{aligned}
& \left|(\alpha, f)_{\phi+\psi}\right|^{2} \\
\leq & \int_{D}|f|_{i \partial \partial \bar{\partial} \psi+\omega}^{2} e^{-(\phi+\psi)} \\
& \times\left(\int_{D} \sum_{j, k=1}^{n} \frac{\partial^{2}(\phi+\psi)}{\partial z_{j} \partial \bar{z}_{k}} \alpha_{j} \bar{\alpha}_{k} e^{-(\phi+\psi)}+\int_{D} \sum_{j, k=1}^{n}\left|\frac{\partial \alpha_{j}}{\partial \bar{z}_{k}}\right|^{2} e^{-(\phi+\psi)}-\int_{D}|\bar{\partial} \alpha|^{2} e^{-(\phi+\psi)}\right) \\
\leq & \int_{D}|f|_{i \partial \partial \bar{\partial} \psi+\omega}^{2} e^{-(\phi+\psi)} \times\left(\int_{D} \sum_{j, k=1}^{n} \frac{\partial^{2}(\phi+\psi)}{\partial z_{j} \partial \bar{z}_{k}} \alpha_{j} \bar{\alpha}_{k} e^{-(\phi+\psi)}+\int_{D} \sum_{j, k=1}^{n}\left|\frac{\partial \alpha_{j}}{\partial \bar{z}_{k}}\right|^{2} e^{-(\phi+\psi)}\right) .
\end{aligned}
$$

Let $f=\sum_{j=1}^{n} f_{j} d \bar{z}_{j}$, set

$$
\left(\alpha_{1}, \alpha_{2}, \cdots, \alpha_{n}\right)=\left(f_{1}, f_{2}, \cdots, f_{n}\right)\left(\frac{\partial^{2} \psi}{\partial z_{j} \partial \bar{z}_{k}}+g_{j \bar{k}}\right)_{n \times n}^{-1},
$$

i.e.,

$$
\left(f_{1}, f_{2}, \cdots, f_{n}\right)=\left(\alpha_{1}, \alpha_{2}, \cdots, \alpha_{n}\right)\left(\frac{\partial^{2} \psi}{\partial z_{j} \partial \bar{z}_{k}}+g_{j \bar{k}}\right)_{n \times n} .
$$

Then inequality (2) becomes

$$
\begin{aligned}
& \left(\int_{D} \sum_{j, k=1}^{n}\left(\frac{\partial^{2} \psi}{\partial z_{j} \partial \bar{z}_{k}}+g_{j \bar{k}}\right) \alpha_{j} \bar{\alpha}_{k} e^{-(\phi+\psi)}\right)^{2} \\
\leq & \int_{D} \sum_{j, k=1}^{n}\left(\frac{\partial^{2} \psi}{\partial z_{j} \partial \bar{z}_{k}}+g_{j \bar{k}}\right) \alpha_{j} \bar{\alpha}_{k} e^{-(\phi+\psi)}\left(\int_{D} \sum_{j, k=1}^{n} \frac{\partial^{2}(\phi+\psi)}{\partial z_{j} \partial \bar{z}_{k}} \alpha_{j} \bar{\alpha}_{k} e^{-(\phi+\psi)}+\int_{D} \sum_{j, k=1}^{n}\left|\frac{\partial \alpha_{j}}{\partial \bar{z}_{k}}\right|^{2} e^{-(\phi+\psi)}\right) .
\end{aligned}
$$

Therefore, we can get

$$
\int_{D} \sum_{j, k=1}^{n}\left(\frac{\partial^{2} \phi}{\partial z_{j} \partial \bar{z}_{k}}-g_{j \bar{k}}\right) \alpha_{j} \bar{\alpha}_{k} e^{-(\phi+\psi)}+\int_{D} \sum_{j, k=1}^{n}\left|\frac{\partial \alpha_{j}}{\partial \bar{z}_{k}}\right|^{2} e^{-(\phi+\psi)} \geq 0 .
$$


We argue by contradiction. Suppose that $i \partial \bar{\partial} \phi-\omega$ is not a semipositive $(1,1)$-form on $D$, then there is $z_{0} \in D, r>0$, a constant $c>0$, and $\xi=\left(\xi_{1}, \xi_{2}, \cdots, \xi_{n}\right) \in \mathbb{C}^{n}$ with $|\xi|=1$, such that

$$
\sum_{j, k=1}^{n}\left(\frac{\partial^{2} \phi}{\partial z_{j} \partial \bar{z}_{k}}-g_{j \bar{k}}(z)\right) \xi_{j} \bar{\xi}_{k}<-c
$$

holds for any $z \in B\left(z_{0}, r\right):=\left\{z \in \mathbb{C}^{n}:\left|z-z_{0}\right|<r\right\} \subset D$. We may assume that $z_{0}=0$, and write $B(0, r)$ as $B_{r}$.

Choose $\chi \in C_{c}^{\infty}\left(B_{r}\right)$, satisfying $\chi(z)=1$ for $z \in B_{r / 2}$. Let $f=\bar{\partial} v$ with

$$
v(z)=\left(\sum_{j=1}^{n} \xi_{j} \bar{z}_{j}\right) \chi(z)
$$

Then

$$
f(z)=\sum_{j=1}^{n} \xi_{j} d \bar{z}_{j}
$$

for $z \in B_{r / 2}$. For $s>0$, set

$$
\psi_{s}(z)=s\left(|z|^{2}-\frac{r^{2}}{4}\right)
$$

It is obvious that $i \partial \bar{\partial} \psi_{s}+\omega>0$ on $\operatorname{supp} f$ for $s \gg 1$. As before, set

$$
\left(\alpha_{1}^{s}, \alpha_{2}^{s}, \cdots, \alpha_{n}^{s}\right)=\left(f_{1}, f_{2}, \cdots, f_{n}\right)\left(\frac{\partial^{2} \psi_{s}}{\partial z_{j} \partial \bar{z}_{k}}+g_{j \bar{k}}\right)^{-1}=\frac{1}{s}\left(\delta_{j k}+\frac{g_{j \bar{k}}}{s}\right)^{-1}\left(f_{1}, f_{2}, \cdots, f_{n}\right) .
$$

We now estimate the integrations on the left hand side of (3) on $B_{r / 2}$ and $D \backslash B_{r / 2}$ separately, with $\alpha$ and $\psi$ replaced by $\alpha^{s}$ and $\psi_{s}$ respectively.

- On $B_{r / 2}$, as $s \rightarrow+\infty$, we have

$$
\alpha^{s}(z)=\frac{1}{s} \sum_{j=1}^{n} \xi_{j} d \bar{z}_{j}+o\left(\frac{1}{s}\right)
$$

and

$$
\frac{\partial \alpha_{j}^{s}}{\partial \bar{z}_{k}}(z)=o\left(\frac{1}{s}\right), j, k=1,2, \cdots, n .
$$

Hence we have

$$
\begin{aligned}
& s^{2} \int_{B_{r / 2}} \sum_{j, k=1}^{n}\left(\frac{\partial^{2} \phi}{\partial z_{j} \partial \bar{z}_{k}}-g_{j \bar{k}}\right) \alpha_{j} \bar{\alpha}_{k} e^{-\left(\phi+\psi_{s}\right)}+s^{2} \int_{B_{r / 2}} \sum_{j, k=1}^{n}\left|\frac{\partial \alpha_{j}}{\partial \bar{z}_{k}}\right|^{2} e^{-\left(\phi+\psi_{s}\right)} \\
& \leq \int_{B_{r / 2}}(-c+o(1)) e^{-\left(\phi+\psi_{s}\right)} .
\end{aligned}
$$


- On $D \backslash B_{r / 2}$, since $f$ has compact support, there is a constant $C>0$, such that $\left|\alpha_{j}^{s}\right| \leq \frac{C}{s}$ and $\left|\frac{\partial \alpha_{j}^{s}}{\partial \bar{z}_{k}}\right| \leq \frac{C}{s}$ hold for $j, k=1,2, \cdots, n$ and $s>0$. Note also that $\lim _{s \rightarrow+\infty} \psi_{s}(z)=+\infty$ for $z \in D \backslash \bar{B}_{r / 2}$. We get

$$
\lim _{s \rightarrow+\infty}\left(s^{2} \int_{D \backslash B_{r / 2}} \sum_{j, k=1}^{n}\left(\frac{\partial^{2} \phi}{\partial z_{j} \partial \bar{z}_{k}}-g_{j \bar{k}}\right) \alpha_{j} \bar{\alpha}_{k} e^{-\left(\phi+\psi_{s}\right)}+s^{2} \int_{D \backslash B_{r / 2}} \sum_{j, k=1}^{n}\left|\frac{\partial \alpha_{j}}{\partial \bar{z}_{k}}\right|^{2} e^{-\left(\phi+\psi_{s}\right)}\right)=0 .
$$

Note that $\psi_{s} \leq 0$ on $B_{r / 2}$, combining equalities (4) and (5) we have,

$$
\int_{D} \sum_{j, k=1}^{n}\left(\frac{\partial^{2} \phi}{\partial z_{j} \partial \bar{z}_{k}}-g_{j \bar{k}}\right) \alpha_{j}^{s} \bar{\alpha}_{k}^{s} e^{-\left(\phi+\psi_{s}\right)}+\int_{D} \sum_{j, k=1}^{n}\left|\frac{\partial \alpha_{j}^{s}}{\partial \bar{z}_{k}}\right|^{2} e^{-\left(\phi+\psi_{s}\right)}<0
$$

for $s>>1$, which contradicts to the inequality (3).

Remark 2.1. From the proof, we can see that in Theorem 2.1, $\psi$ can be taken to be of the form $a|z-w|^{2}+b$, with $a \gg 1$ and $b \in \mathbb{R}$.

Remark 2.2. In Theorem 1.1, we can allow $\phi$ to have poles, with the condition that $\operatorname{Pol}(\phi):=$ $\phi^{-1}(-\infty)$ is closed in $D$, and $\phi$ is upper semi-continuous on $D$, and is $C^{2}$-smooth and satisfies the optimal $L^{2}$-estimate property on $D \backslash \operatorname{Pol}(\phi)$.

2.2. In terms of the multiple coarse $L^{p}$-estimate property. The purpose of this subsection is to prove Theorem 1.3, which is the following

Theorem 2.3 (= Theorem 1.3). Let $D$ be a domain in $\mathbb{C}^{n}$ and $\phi: D \rightarrow \mathbb{R}$ be a continuous function. If $\phi$ satisfies the multiple coarse $L^{p}$-estimate property for some $p>1$, then $\phi$ is plursubharmonic on $D$.

Proof. We prove the theorem by modifying the idea in [13]. We will show that $(D, \phi)$ satisfies the multiple coarse $L^{p}$-extension property. Note that $\left.\phi\right|_{D^{\prime}}$ also satisfies the multiple coarse $L^{p}$-estimate property for any open set $D^{\prime} \subset D$, replacing $D$ by a relatively compact open subset of it, we may assume that $D$ is bounded and $\phi$ is uniformly continuous on $D$.

Fix an integer $m>0$ and $w \in D$. We will construct a holomorphic function $f \in O(D)$ such that $f(w)=1$ and

$$
\int_{D}|f|^{p} e^{-m \phi} \leq C_{m}^{\prime} e^{-m \phi(w)}
$$

where $C_{m}^{\prime}$ is a constant independent of the choice of $w \in D$ satisfying $\lim _{m \rightarrow \infty} \frac{\log C_{m}^{\prime}}{m}=0$.

Let $\chi=\chi(t)$ be a smooth function on $\mathbb{R}$, such that

- $\chi(t)=1$ for $t \leq 1 / 4$,

- $\chi(t)=0$ for $t \geq 1$, and

- $\left|\chi^{\prime}(t)\right| \leq 2$ on $\mathbb{R}$. 
Define a $(0,1)$-form $\alpha_{\epsilon}$ by

$$
\alpha_{\epsilon}:=\bar{\partial} \chi\left(\frac{|z-w|^{2}}{\epsilon^{2}}\right)=\chi^{\prime}\left(\frac{|z-w|^{2}}{\epsilon^{2}}\right) \sum_{j} \frac{z_{j}-w_{j}}{\epsilon^{2}} d \bar{z}_{j},
$$

and set

$$
\psi_{\delta}:=|z|^{2}+n \log \left(|z-w|^{2}+\delta^{2}\right),
$$

where $0<\epsilon \leq 1$ and $\delta$ are positive parameters. From the multiple coarse $L^{p}$-estimate property, we obtain a smooth function $u_{\epsilon, \delta}$ on $D$ such that $\bar{\partial} u_{\epsilon, \delta}=\alpha_{\epsilon}$ and

$$
\int_{D}\left|u_{\epsilon, \delta}\right|^{p} e^{-\left(m \phi+\psi_{\delta}\right)} \leq C_{m} \int_{D}\left|\alpha_{\epsilon}\right|_{i \partial \bar{\partial} \psi_{\delta}}^{p} e^{-\left(m \phi+\psi_{\delta}\right)}
$$

Since

$$
\begin{aligned}
\left|\alpha_{\epsilon}\right|_{\sqrt{-1} \partial \bar{\partial} \psi_{\delta}} & =\left|\chi^{\prime}\left(\frac{|z-w|^{2}}{\epsilon^{2}}\right)\right| \cdot \frac{1}{\epsilon^{2}} \cdot\left|\sum_{j}\left(z_{j}-w_{j}\right) d \bar{z}_{j}\right|_{i \partial \partial \bar{\partial} \psi_{\delta}} \\
& \leq\left|\chi^{\prime}\left(\frac{|z-w|^{2}}{\epsilon^{2}}\right)\right| \cdot \frac{1}{\epsilon^{2}} \cdot\left|\sum_{j}\left(z_{j}-w_{j}\right) d \bar{z}_{j}\right|_{i \partial \partial \bar{\partial}|z|^{2}} \\
& =\left|\chi^{\prime}\left(\frac{|z-w|^{2}}{\epsilon^{2}}\right)\right| \cdot \frac{1}{\epsilon^{2}}|z-w|,
\end{aligned}
$$

the support of $\chi^{\prime}\left(\frac{|z-w|^{2}}{\epsilon^{2}}\right)$ is in $\left\{1 / 4 \leq|z-w|^{2} / \epsilon^{2} \leq 1\right\}$, and $\psi_{\delta} \geq 2 n \log |z-w|$, we have that

$$
\begin{aligned}
\text { (RHS of (6)) } & \leq C_{m} \int_{\left\{\epsilon^{2} / 4 \leq|z-w|^{2} \leq \epsilon^{2}\right\}}\left|\chi^{\prime}\left(\frac{|z-w|^{2}}{\epsilon^{2}}\right)\right|^{p} \frac{1}{\epsilon^{2 p}}|z-w|^{p} e^{-\left(m \phi+\psi_{\delta}\right)} \\
& \leq C_{m} \frac{2^{p}}{\epsilon^{2 p}} \int_{\left\{\epsilon^{2} / 4 \leq|z-w|^{2} \leq \epsilon^{2}\right\}}|z-w|^{p} e^{-\left(m \phi+\psi_{\delta}\right)} \\
& \leq C_{m} \frac{2^{p}}{\epsilon^{2 p}} \int_{\left\{\epsilon^{2} / 4 \leq|z-w|^{2} \leq \epsilon^{2}\right\}} \epsilon^{p} e^{-m \inf _{B(w, \epsilon)} \phi} e^{-2 n \log |z-w|} \\
& \leq C C_{m} \frac{e^{-m \inf _{B(w, \epsilon)} \phi}}{\epsilon^{p}},
\end{aligned}
$$

where $C=2^{p+2 n} \mu\left(B_{1}\right)$.

To summarize, we have obtained a smooth function $u_{\epsilon, \delta}$ on $D$ such that

- $\bar{\partial} u_{\epsilon, \delta}=\alpha_{\epsilon}$, and

- the following estimate holds:

$$
\int_{D}\left|u_{\epsilon, \delta}\right|^{p} e^{-\left(m \phi+\psi_{\delta}\right)} \leq C C_{m} \frac{e^{-m \inf _{\mathbf{B}(w, \epsilon)} \phi}}{\epsilon^{p}} .
$$

Note that the weight function $\psi_{\delta}$ is decreasing when $\delta \searrow 0, e^{-\psi_{\delta}}$ is increasing when $\delta \searrow 0$. Fix $\delta_{0}>0$. Then, for $\delta<\delta_{0}$, we have that

$$
\int_{D}\left|u_{\epsilon, \delta}\right|^{p} e^{-\left(m \phi+\psi_{\delta_{0}}\right)} \leq \int_{D}\left|u_{\epsilon, \delta}\right|^{p} e^{-\left(m \phi+\psi_{\delta}\right)} \leq C C_{m} \frac{e^{-m \inf _{B(w, \epsilon)} \phi}}{\epsilon^{p}} .
$$


Thus $\left\{u_{\epsilon, \delta}\right\}_{\delta<\delta_{0}}$ forms a bounded sequence in $L^{p}\left(D, e^{-\left(m \phi+\delta_{0}\right)}\right)$. Note that $p>1$, we can choose a sequence $\left\{u_{\epsilon, \delta^{(k)}}\right\}_{k}$ in $L^{p}\left(D, e^{-\left(m \phi+\delta_{0}\right)}\right)$ which weakly converges to some $u_{\epsilon} \in L^{p}\left(D, e^{-\left(m \phi+\delta_{0}\right)}\right)$, satisfying

$$
\int_{D}\left|u_{\epsilon}\right|^{p} e^{-\left(m \phi+\psi_{\delta_{0}}\right)} \leq C C_{m} \frac{e^{-m \inf _{B(w, \epsilon)} \phi}}{\epsilon^{p}} .
$$

Repeating this argument for a sequence $\left\{\delta_{j}\right\}$ decreasing to 0 , and by diagonal argument, we can select a sequence $\left\{u_{\epsilon, \delta^{k}}\right\}_{k}$ which weakly converges to $u_{\epsilon}$ in $L^{p}\left(D, e^{-\left(m \phi+\psi_{\delta_{j}}\right)}\right)$ and $u_{\epsilon}$ satisfies estimates

$$
\int_{D}\left|u_{\epsilon}\right|^{p} e^{-\left(m \phi+\psi_{\delta_{j}}\right)} \leq C C_{m} \frac{e^{-m \inf _{B(w, \epsilon)} \phi}}{\epsilon^{p}}
$$

for all $j$. By the monotone convergence theorem,

$$
\int_{D}\left|u_{\epsilon}\right|^{p} e^{-\left(m \phi+\psi_{0}\right)} \leq C C_{m} \frac{e^{-m \inf _{B(w, \epsilon)} \phi}}{\epsilon^{p}} .
$$

Since $\bar{\partial}$ is weakly continuous, we also have $\bar{\partial} u_{\epsilon}=\alpha_{\epsilon}$.

Since $\frac{1}{|z-w|^{2 n}}$ is not integrable near $w, u_{\epsilon}(w)$ must be 0 . Let $f_{\epsilon}:=\chi\left(|z-w|^{2} / \epsilon^{2}\right)-u_{\epsilon}$. Then $f_{\epsilon} \in O(D), f_{\epsilon}(0)=1$ and

$$
\begin{aligned}
\int_{D}\left|f_{\epsilon}\right|^{p} e^{-m \phi} & \leq\left(\left(\left.\int_{D} \chi\left(\frac{|z-w|^{2}}{\epsilon^{2}}\right)\right|^{p} e^{-m \phi}\right)^{1 / p}+\left(\int_{D}\left|u_{\epsilon}\right|^{p} e^{-m \phi}\right)^{1 / p}\right)^{p} \\
& \leq 2^{p}\left(\int_{D}\left|\chi\left(\frac{|z-w|^{2}}{\epsilon^{2}}\right)\right|^{p} e^{-m \phi}+\int_{D}\left|u_{\epsilon}\right|^{p} e^{-m \phi}\right)
\end{aligned}
$$

Since $\chi \leq 1$ and the support of $\chi\left(|z-w|^{2} / \epsilon^{2}\right)$ is contained in $\left\{|z-w|^{2} \leq \epsilon^{2}\right\}$ and $0<\epsilon \leq 1$, we have

$$
\int_{D}\left|\chi\left(\frac{|z-w|^{2}}{\epsilon^{2}}\right)\right|^{p} e^{-m \phi} \leq \mu\left(B_{1}\right) e^{-m \inf _{B(w, \epsilon)} \phi}
$$

We also have

$$
\begin{aligned}
\int_{D}\left|u_{\epsilon}\right|^{p} e^{-m \phi} & \leq \sup _{z \in D} e^{\psi_{0}(z)} \cdot \int_{D}\left|u_{\epsilon}\right|^{p} e^{-\left(m \phi+\psi_{0}\right)} \\
& \leq \sup _{z \in D} e^{\psi_{0}(z)} \cdot C C_{m} \frac{e^{-m \inf _{B(w, \epsilon)} \phi}}{\epsilon^{p}} \\
& \leq C^{\prime} C_{m} \frac{e^{-m \inf _{B(w, \epsilon)} \phi}}{\epsilon^{p}}
\end{aligned}
$$

where $C^{\prime}$ is a constant depends only on the diameter of $D$. We may assume $C_{m} \geq 1$. Combining these estimates with (9), we obtain that

$$
\int_{D}\left|f_{\epsilon}\right|^{p} e^{-m \phi} \leq C^{\prime \prime} C_{m} \frac{1}{\epsilon^{p}} e^{-m \inf _{B(w, \epsilon)} \phi},
$$

where $C^{\prime \prime}$ is a constant independent of $m$ and $w$.

Let

$$
O_{\epsilon}=\sup _{z, w \in D,|z-w| \leq \epsilon}|\phi(z)-\phi(w)| .
$$


By the uniform continuity of $\phi, O_{\epsilon}$ is finite and goes to 0 as $\epsilon \rightarrow 0$. Let $\epsilon:=1 / m$. We have $|m \phi(z)-m \phi(w)| \leq m O_{1 / m}$ for $|z-w| \leq 1 / m$. Then

$$
\begin{aligned}
\int_{D}\left|f_{1 / m}\right|^{p} e^{-m \phi} & \leq C^{\prime \prime} C_{m} m^{p} e^{-m \phi(w)+m O_{1 / m}} \\
& =C^{\prime \prime} C_{m} m^{p} e^{m O_{1 / m}} e^{-m \phi(w)} .
\end{aligned}
$$

Let $C_{m}^{\prime}=C^{\prime \prime} C_{m} m^{p} e^{m O_{1 / m}}$, we have

$$
\frac{\log C_{m}^{\prime}}{m}=\frac{\log \left(C^{\prime \prime} C_{m} m^{p}\right)}{m}+O_{1 / m} \rightarrow 0
$$

which implies $\phi$ satisfies the multiple coarse $L^{p}$-extension property on $D$, and hence $\phi$ is plurisubharmonic by Theorem 1.5.

Remark 2.3. In Theorem 2.3, we can allow $\phi$ to have poles, with the condition that $\operatorname{Pol}(\phi):=$ $\phi^{-1}(-\infty)$ is closed in $D$, and $\phi$ is upper semi-continuous on $D$, and is continuous and satisfies the multiple coarse $L^{p}$-estimate property on $D \backslash \operatorname{Pol}(\phi)$.

\section{Characterizations OF PlURISUbHaRMONIC FUNCTIONS IN TERMS OF $L^{p}$-EXTENSIONS OF HOLOMORPHIC} FUNCTIONS

In this section, we discuss characterizations of plurisubharmonic functions in terms of $L^{p}$-extensions of holomorphic functions. The aim is to prove Theorem 1.4 and 1.5. The idea is inspired by GuanZhou's method in [10].

We first prove a lemma as follows.

Lemma 3.1. Let $D \subset \mathbb{C}^{n}$ be a domain, and $\phi$ be an upper semi-continuous function on $D$. Then $\phi$ is plurisubharmonic if and only if for any $z_{0} \in D$ and any holomorphic cylinder $P$ with with $z_{0}+P \subset \subset D$

$$
\phi\left(z_{0}\right) \leq \frac{1}{\mu(P)} \int_{z_{0}+P} \phi .
$$

Proof. The "only if" part follows easily from the mean value inequality for plurisubharmonic functions. We now give the proof of the "if" part.

For any point $z_{0} \in D$, any $\xi \in \mathbb{C}^{n}$ with $|\xi|=1$, and $r>0$ such that $\left\{z_{0}+\zeta_{1} \xi:\left|\zeta_{1}\right| \leq r_{1}\right\} \subset D$. Choose an orthonormal basis $f_{1}, f_{2}, \cdots, f_{n}$ of $\mathbb{C}^{n}$ with $f_{1}=\xi$. There is $s_{0}>0$ such that $z_{0}+\zeta_{1} \xi+\sum_{j=2}^{n} \zeta_{j} f_{j} \in$ $D$ for all $\left|\zeta_{1}\right| \leq r$ and $\sum_{j=2}^{n}\left|\zeta_{j}\right|^{2} \leq s_{0}^{2}$. Let $A=\left(f_{1}, f_{2}, \cdots, f_{n}\right)$, then $z_{0}+A\left(P_{r, s}\right) \subset \subset D$, for $s<s_{0}$. By assumption, we have

$$
\begin{aligned}
\phi\left(z_{0}\right) & \leq \frac{1}{\mu\left(A\left(P_{r, s}\right)\right)} \int_{z_{0}+A\left(P_{r, s}\right)} \phi \\
& =\frac{1}{\mu\left(P_{r, 1}\right)} \int_{P_{r, 1}} \phi\left(z_{0}+\zeta_{1} \xi+s \sum_{j=2}^{n} \zeta_{j} f_{j}\right) .
\end{aligned}
$$


As $\phi$ is upper semi-continuous on $D$ and $\left\{z_{0}+\zeta_{1} \xi:\left|\zeta_{1}\right| \leq r\right\}$ is compact, we may assume $\phi \leq 0$ on $z_{0}+A\left(P_{r, s_{0}}\right)$. Then we have

$$
\begin{aligned}
\phi\left(z_{0}\right) & \leq \frac{1}{\mu\left(P_{r, 1}\right)} \int_{P_{r, 1}} \limsup _{s \rightarrow 0} \phi\left(z_{0}+\zeta_{1} \xi+s \sum_{j=2}^{n} \zeta_{j} f_{j}\right) \\
& \leq \frac{1}{\mu\left(P_{r, 1}\right)} \int_{P_{r, 1}} \phi\left(z_{0}+\zeta_{1} \xi\right) \\
& =\frac{1}{\pi r^{2}} \int_{\left\{\zeta_{1} \in \mathbb{C}:\left|\zeta_{1}\right|<r\right\}} \phi\left(z_{0}+\zeta_{1} \xi\right),
\end{aligned}
$$

where the first inequality follows from (11) and Fatou's lemma, the second inequality follows from the fact that $\phi$ is upper semi-continuous, and the last equality holds since $\phi\left(z_{0}+\zeta_{1} \xi\right)$ is independent of $\zeta_{2}, \cdots, \zeta_{n}$.

Theorem 3.2 (= Theorem 1.4). Let $\phi: D \rightarrow[-\infty,+\infty)$ be an upper semi-continuous function on a domain $D$ in $\mathbb{C}^{n}$. If $\phi$ satisfies the optimal $L^{p}$-extension property for some $p>0$, then $\phi$ is plurisubharmonic on $D$.

Proof. Take arbitrary $z_{0} \in D$, such that $\phi\left(z_{0}\right) \neq-\infty$. For any holomorphic cylinder $P$ with $z_{0}+P \subset D$, by the optimal $L^{p}$-extension property and taking $\log$, we get that

$$
\phi\left(z_{0}\right) \leq-\log \left(\frac{1}{\mu(P)} \int_{z_{0}+P}|f|^{p} e^{-\phi}\right) .
$$

By Jensen's inequality, we have

$$
\begin{aligned}
\phi\left(z_{0}\right) & \leq \frac{1}{\mu(P)} \int_{z_{0}+P}-\log \left(|f|^{p} e^{-\phi}\right) \\
& =\frac{1}{\mu(P)} \int_{z_{0}+P} \phi-\frac{1}{\mu(P)} \int_{z_{0}+P} p \log |f| .
\end{aligned}
$$

Note that $p \log |f|$ is a plurisubharmonic function and $f\left(z_{0}\right)=1$, by Fubini's theorem, we have

$$
-\frac{1}{\mu(P)} \int_{z_{0}+P} p \log |f| \leq 0 .
$$

Therefore, we get the desired mean-value inequality,

$$
\phi\left(z_{0}\right) \leq \frac{1}{\mu(P)} \int_{z_{0}+P} \phi .
$$

By Lemma 3.1, $\phi$ is plurisubharmonic on $D$.

Theorem 3.3 (= Theorem 1.5). Let $\phi: D \rightarrow[-\infty,+\infty)$ be an upper semi-continuous function on a domain $D$ in $\mathbb{C}^{n}$. If $\phi$ satisfies the multiple coarse $L^{p}$-extension property for some $p>0$, then $\phi$ is plursubharmonic on $D$. 
Proof. Let $z_{0} \in D$ such that $\phi\left(z_{0}\right) \neq-\infty$, and let $P$ be a holomorphic cylinder with $z_{0}+P \subset \subset D$. By assumption, for any $m \geq 1$, there is a holomorphic function $f_{m}$ on $D$, with $f_{m}\left(z_{0}\right)=1$ and

$$
\int_{z_{0}+P}\left|f_{m}\right|^{p} e^{-m \phi} \leq \int_{D}\left|f_{m}\right|^{p} e^{-m \phi} \leq C_{m} e^{-m \phi(z)} .
$$

Dividing both sides by $\mu(P)$ and taking log, we get

$$
\log \frac{1}{\mu(P)} \int_{z_{0}+P}\left|f_{m}\right|^{p} e^{-m \phi} \leq \log \frac{C_{m}}{\mu(P)}-m \phi\left(z_{0}\right) .
$$

Then we have

$$
\phi\left(z_{0}\right) \leq \frac{\log C_{m}}{m}-\frac{\log \mu(P)}{m}-\frac{1}{m} \log \frac{1}{\mu(P)} \int_{z_{0}+P}\left|f_{m}\right|^{p} e^{-m \phi}
$$

By Jensen's inequality and

$$
\begin{aligned}
& -\frac{1}{m} \log \left(\frac{1}{\mu(P)} \int_{z_{0}+P}\left|f_{m}\right|^{p} e^{-m \phi}\right) \\
\leq & -\frac{1}{m} \frac{1}{\mu(P)} \int_{z_{0}+P} \log \left(\left|f_{m}\right|^{p} e^{-m \phi}\right) \\
= & -\frac{1}{m} \frac{1}{\mu(P)} \int_{z_{0}+P} p \log \left|f_{m}\right|+\frac{1}{\mu(P)} \int_{z_{0}+P} \phi .
\end{aligned}
$$

Note that $p \log \left|f_{m}\right|$ is a plurisubharmonic function, and $f_{m}\left(z_{0}\right)=1$, the first term in the above equality is nonpositive, therefore,

$$
-\frac{1}{m} \log \left(\frac{1}{\mu(P)} \int_{z_{0}+P}\left|f_{m}\right|^{p} e^{-m \phi}\right) \leq \frac{1}{\mu(P)} \int_{z_{0}+P} \phi .
$$

Combining inequalities (12) and (13), we get

$$
\phi\left(z_{0}\right) \leq \frac{\log C_{m}}{m}-\frac{\log \mu(P)}{m}+\frac{1}{\mu(P)} \int_{z_{0}+P} \phi .
$$

As $\lim _{m \rightarrow \infty} \frac{\log C_{m}}{m}=0$ and $\lim _{m \rightarrow \infty} \frac{\log \mu(P)}{m}=0$, taking limit, we have

$$
\phi\left(z_{0}\right) \leq \frac{1}{\mu(P)} \int_{z_{0}+P} \phi .
$$

From Lemma 3.1, $\phi$ is plurisubharmonic on $D$.

\section{REFERENCES}

[1] B. Berndtsson. $L^{2}$-methods for the $\bar{\partial}$-equation, enotes, http://www.math.chalmers.se/ bob/not3.pdf. 2, 3

[2] B. Berndtsson. Prekopa's theorem and Kiselman's minimum principle for plurisubharmonic functions. Math. Ann., 312(4):785-792, 1998. 2

[3] B. Berndtsson. Subharmonicity properties of the Bergman kernel and some other functions associated to pseudoconvex domains. Ann. Inst. Fourier (Grenoble), 56(6):1633-1662, 2006. 3 
[4] B. Berndtsson. Curvature of vector bundles associated to holomorphic fibrations. Ann. of Math. (2), 169(2):531$560,2009.3$

[5] Z. Błocki. Suita conjecture and the Ohsawa-Takegoshi extension theorem. Invent. Math., 193(1):149-158, 2013. 2

[6] J.-P. Demailly. Regularization of closed positive currents and intersection theory. J. Algebraic Geom., 1(3):361409, 1992.3

[7] J.-P. Demailly. Analytic methods in algebraic geometry, volume 1 of Surveys of Modern Mathematics. International Press, Somerville, MA; Higher Education Press, Beijing, 2012. 4

[8] F. Deng, Z. Wang, L. Zhang, and X. Zhou. New characterization of plurisubharmonic functions and positivity of direct image sheaves. arXiv:1809.10371.2, 3, 4

[9] Q. Guan and X. Zhou. Optimal constant problem in the $L^{2}$ extension theorem. C. R. Math. Acad. Sci. Paris, 350(15-16):753-756, 2012. 2

[10] Q. Guan and X. Zhou. A solution of an $L^{2}$ extension problem with an optimal estimate and applications. Ann. of Math. (2), 181(3):1139-1208, 2015. 2, 3, 11

[11] C. Hacon, M. Popa, and C. Schnell. Algebraic fiber spaces over abelian varieties: Around a recent theorem by Cao and Păun. In Local and global methods in algebraic geometry, volume 712 of Contemp. Math., pages 143-195. Amer. Math. Soc., Providence, RI, 2018. 2

[12] L. Hörmander. $L^{2}$ estimates and existence theorems for the $\bar{\partial}$ operator. Acta Math., 113:89-152, 1965. 1, 5

[13] G. Hosono and T. Inayama. A converse of Hörmander's $L^{2}$-estimate and new positivity notions for vector bundles. arXiv:1901.02223v1.2, 3, 8

[14] T. Ohsawa and K. Takegoshi. On the extension of $L^{2}$ holomorphic functions. Math. Z., 195(2):197-204, 1987. 1, 2

Fusheng Deng: School of Mathematical Sciences, University of Chinese Academy of Sciences, Beijing 100049, P. R. CHINA

E-mail address: fshdeng@ucas.ac.cn

Jiafu Ning: Department of Mathematics, Central South University, Changsha, Hunan 410083, P. R. China.

E-mail address: jfning@csu.edu.cn

Zhiwei Wang: School of Mathematical Sciences, Beijng Normal University, Beijng, 100875, P. R. China

E-mail address: zhiwei@bnu.edu.cn 